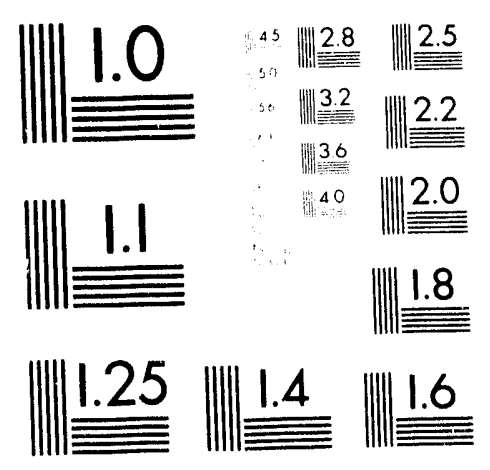



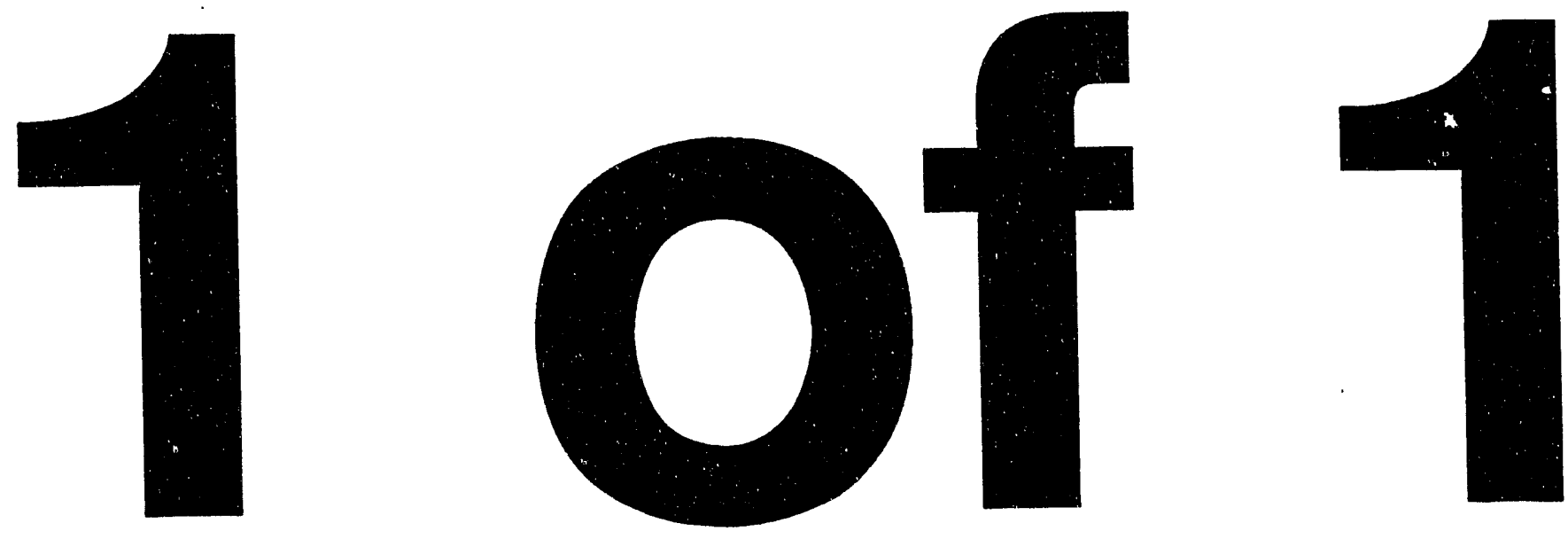

11 


\title{
Third-Order Corrections to the SLC Final Focus.*
}

\author{
N. J. Walker, R. Helm, J. Irwin, M. Woodley. \\ Stanford Linear Accelerator Center, Stanford University; Stanford, California 94309
}

\begin{abstract}
The minimum $\beta$ achievable at the interaction point $\left(\beta^{*}\right)$ with the current design of the SLC final focus is limited to $\sim 5 \mathrm{~mm}$ by third order optical aberrations, most notably the $\mathrm{U}_{1266}$ and $\mathrm{U}_{3466}$ terms (using the notation of $\mathrm{K}$. Brown). A new lattice is presented which effectively zeros these terms. The remaining third order terms which accrue from the interleaved sextupole pairs in the chromatic correction section (CCS) can be cancelled by the inclusion of five octupoles (two in the CCS, and three in the final telescope). The resulting final focus system is corrected to third order for any usable range of $\beta^{*}$ (given the constraints on the beam divergence at the interaction point). The potential luminosity obtainable from such a system is also presented.
\end{abstract}

\section{INTRODUCTION.}

The current design of the SLC final focus essentially follows the design proposed by Brown[1]. It consists of two telescope sections, separated by a chromatic correction section (CCS) where two strong interleaved sextupole pairs ( $\mathrm{X}$ and $\mathrm{Y}$ ) are used to cancel the chromatic contribution to the beam size at the interaction point (IP)[2]. Although the design effectively cancels the second-order (chromatic) aberrations, the bandwidth of the system is limited by third-order effects. Analysis of these aberrations[3] has shown that they arise from two separate sources: (i) a small phase error between the sextupole pairs and the final triplet, and (ii) the interleaved $X$ and $Y$ sextupole pairs. The effect of the aberrations is to limit the minimum: $\beta^{*}$ at the IP to approximately $5 \mathrm{~mm}$ in both planes; a smaller $\beta^{*}$ leads to a larger beam size as the aberrations begin to dominate over the linear optics. With the typical beam emittances seen in the final focus during the 1992 physics run ( 600 by $400 \mu \mathrm{m} \mu \mathrm{r}$ in the horizontal and vertical planes respectively), the smallest possible $\beta^{*}$ was limited by SLD background considerations to give a beam divergence at the IP $\left(\theta^{*}\right)$ of $-300 \mu \mathrm{r}$ in both planes, corresponding to $\mathrm{a} \beta^{*}{ }_{x}$ of $\sim 7 \mathrm{~mm}$ and a $\beta^{*}$ y of $\sim 5 \mathrm{~mm}$, which compare well to the optical optimum. For the current (1993) physics run, a "flat beam" is being used[4], where the vertical emittance in the final focus is of the order of $60 \mu \mathrm{m} \mu \mathrm{r}$. The smallest vertical spot size $(\sim 0.8 \mu \mathrm{m})$ is now achieved with an IP beam divergence of approximately $100 \mu$, well below the SLD background limit. In order to increase the divergence (decrease the $\beta^{*}$ ), it is first necessary to identify and correct those aberrations which limit the beam

\footnotetext{
* Work supported by Department of Energy contract DE-AC03-76SFOO515
}

size. In this paper, a third-order correction scheme is proposed which effectively minimizes the third-order contributions to the beam size, enabling a vertical optimal $\beta^{*}$ of $\sim 1 \mathrm{~mm}$, achieving a vertical spot size of $\sim 0.3 \mu \mathrm{m}$ at a divergence of $245 \mu \mathrm{r}$, given a vertical emittance of $60 \mu \mathrm{r} \mu \mathrm{m}$. The decreased spot size gives a luminosity increase by a factor of 2.8 from the geometry alone, and an additional factor of -1.4 from the beam-beam interaction (pinch effect), giving an overall luminosity gain of a factor of approximate $\times 4$.

\section{LIMITING THIRD-ORDER ABERRATIONS.}

An analysis of the SLC final focus has shown[1] that the dominant vertical aberration is a high order chromaticity, $\mathrm{U}_{3466}$ (using the TRANSPORT[5] matrix notation). Using Lie Algebra techniques a complete analysis of the important third(optical) order aberrations has been made in terms of monomials in the Hamiltonian[3]. The monomial corresponding to the afore mentioned chromaticity is $y^{\prime 2} \delta^{2}$, where $y^{\prime}$ is taken to be the phase space coordinate at the IP. In all furher discussions, it is assumed that the phase space coordinates $\left(x, x^{\prime}, y, y^{\prime}\right)$ refer to those at the interaction point. Table 1 gives the results of the analysis (taken from [3]).

\begin{tabular}{cccc}
\hline Monomial & $\begin{array}{c}\text { TRANSPORT } \\
\text { notation }\end{array}$ & $\begin{array}{c}\text { Cocfficient } \\
\text { (metcrs) }\end{array}$ & $\begin{array}{c}\text { \% of total } \\
\sigma^{2}\end{array}$ \\
\hline$y^{\prime 2} \delta^{2}$ & $\mathrm{U}_{3466}$ & 229.5 & 86 \\
$x^{\prime} y^{2} \delta$ & $\mathrm{U}_{1446} / \mathrm{U}_{3246}$ & 817.5 & 6 \\
linear & & & 3 \\
$x^{\prime 2} y^{2}$ & $\mathrm{U}_{1244} / \mathrm{U}_{3224}$ & -2861.7 & 2.2 \\
$x^{\prime} \delta^{2}$ & $\mathrm{U}_{1466} \mathrm{U}_{3266}$ & 55.5 & 1.9 \\
\hline
\end{tabular}

Table 1. Most significant aberrations to ${\sigma^{\circ}}_{y}$ in order of contritition

$$
\left(\theta^{*}=245 \mu r\right)
$$

The vertical beam size is dominated by the $y^{2} \delta^{2}$ term. Further analysis of this aberration[3] show's that it is almost entirely due to the interaction of the sextupoles and the triplet chromaticity. If the sextupole pair are at exactly the same phase as the triplet ( $\pi / 2$ out of phase with the IP), then the chromaticity from the sextupoles is simply given by $-K_{s} \eta_{s} R_{3-4}^{2} y^{\prime 2} \delta$. where $K_{s}$ is the integrated sextupole strength, $\eta_{s}$ the dispersion function at the sextupoles, and $R_{34}$ is the linear Green's function from the sextupole to the IP. The sextupole strength is then adjusted to exactly cancel the total chromaticity of the system In the current SLC design, however, the sextupoles are not exactly at the correct phase, and the expression for the sextupole chromaticity (with respect to the IP) is given by

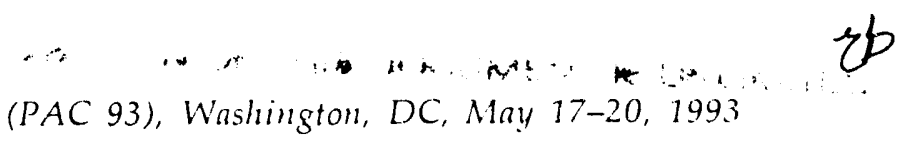




$$
\begin{aligned}
& -K_{s} \eta_{s}\left(R_{44} y-R_{34} y^{\prime}\right)^{2} \delta \\
= & -K_{s} \eta_{s} R_{34}^{2} y^{\prime 2} \delta \\
& +2 K_{s} \eta_{s} R_{34} R_{44} y y^{\prime} \delta \\
& -K_{s} \eta_{s} R_{44}^{2} y^{2} \delta
\end{aligned}
$$

The first term in (2) is the desired chromaticity term which remains unchanged. The last term does not contribute to the vertical beam size at the IP. The contribution to the beam size from the second ( $y$ ' $\delta$ ) term is also small; however, this phase can interact with the triplet chromaticity $\left(\xi_{2} y^{\prime 2} \delta\right)$ to give the $y^{\prime 2} \delta^{2}$ term, which can be estimated by taking the poisson bracket of the two moncmials[3]:

$$
\frac{1}{2}\left[2 K_{s} \eta_{s} R_{34} R_{44} y y^{\prime} \delta, \xi y^{\prime 2} \delta\right]=2 K_{s} \eta_{s} R_{34} R_{44} \xi y^{\prime 2} \delta^{2} .
$$

Since the triplet chromaticity is approximately $60 \%$ of the total chromaricity of the final focus, we can replace $\xi$ by $\xi \approx 0.6 \times K_{s} \eta_{s} R_{34}^{2}$, and the right hand side of (2) becomes

$$
1.2 \times K_{s}^{2} \eta_{s}^{2} R_{34}^{3} R_{44} y^{\prime 2} \delta^{2}
$$

For the current design final focus, equation 3 gives a coefficient for the $y^{\prime 2} \delta^{2}$ term of $200 \mathrm{~m}$, which is within $15 \%$ of the total coefficient given in table 1 .

\section{CORRECTION OF $y^{\prime 2} \delta^{2}\left(\mathrm{U}_{3466}\right)$ TERM.}

$R_{34}$ and $R_{44}$ are in effect the $\mathrm{Y}$ sine-like and cosine-like rays respectively, traced backwards from the IP. The sine-like ray drives the $\beta$ functions and chromaticity of the final focus, and is the dominant (magnified) phase. The cosine-like ray is the demagnified phase. Equation 3 shows that the $y^{\prime 2} \delta^{2}$ term depends on the value of the cosine-like ray at the $Y$ sextupoles $\left(R_{4,4}\right)$. By adjusting the optics of the final telescope using a new quadrupole placed close to the IP image point, it is possible to adjust the position of the cosine-like ray at the $Y$ sextupoles, while leaving the sine-like ray ( $\beta$ functions) unchanged (figure 1).

An ideal location for the new quadrupole is exactly at the image point at the entrance to the final telescope: here, the sine-like ray passes naturally through zero and is unaffected by the quadrupole. Unfortunately, the image point is at the center of the B1 dipole, and a less optimal solution had to be found requiring the adjustment of the other magnets in the final telescope to correct the resulting perturbations to the $\beta$ functions. In addition, it was also discovered advantageous to reflect the symmetry of the CCS optical functions (focusing magnets become defocusing and visa versa), to allow the new quadrupole to drive the cosine-iike ray through zero at the $Y$ sextupoles. Adjustment of the value of the cosine-like ray at the $Y$ sextupoles (and hence the $R_{44}$ ), allows tuning of the $y^{\prime 2} \delta^{2}$ term. The final solution does not have the cosine-like ray passing though the center of the $\mathrm{Y}$ sextupoles, but rather the ray is offset in order to cancel the remaining 15-20\% of the aberration coming from other sources in the final focus. The solution also
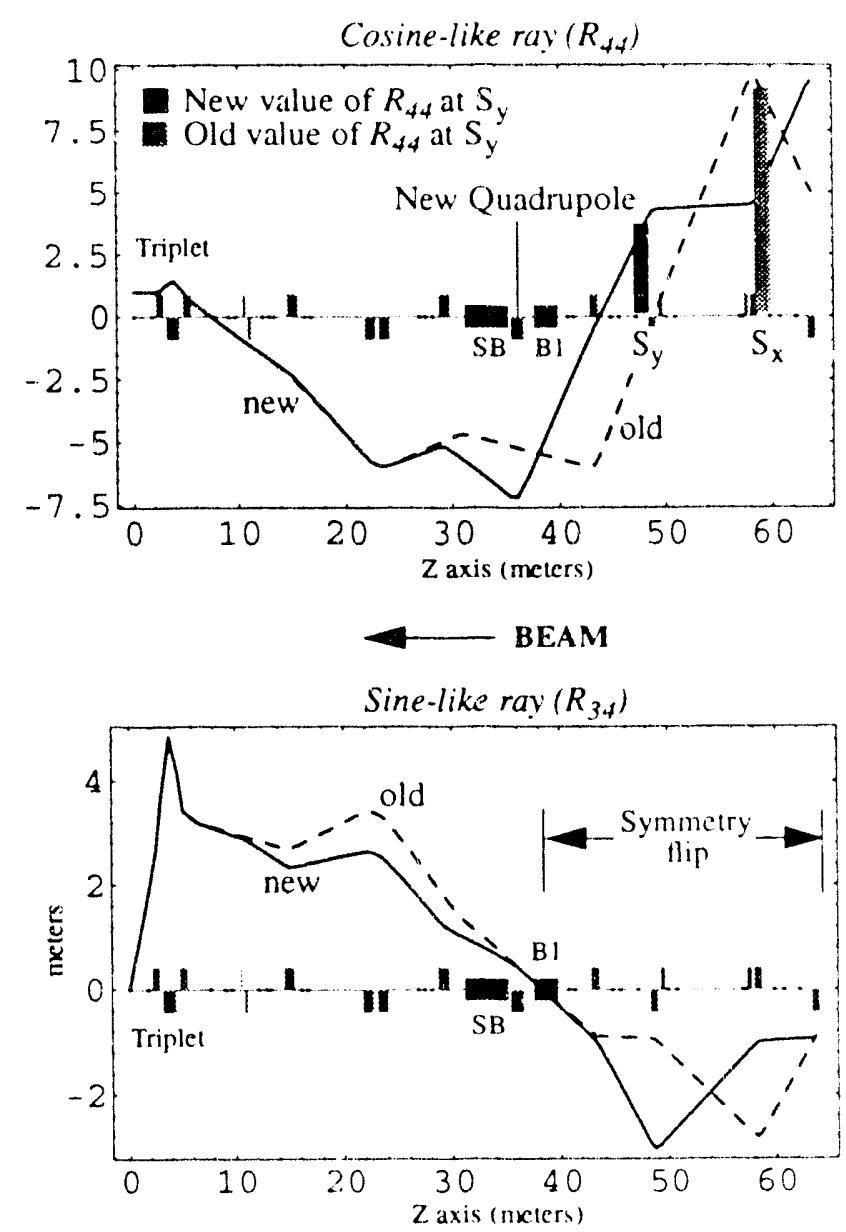

Figure 1. Sine- and cosine-like rays traced hackwards from the IP through the final telescope and part of the CCS. Solid line represents the proposed (new) optics, while the dashed line represents the current (old) optics. On the top graph (cosine-like), the old and new tal. ues of the ray at the $Y$ sextupole are indicated hy the vertical hars (Note: hecause of the symmetry exchange in the CCS the $X$ and sexupoles have interchanged)

minimizes the $x^{2} \delta^{2}$ aberration $\left(\mathrm{U}_{1260}\right)$ to the horizontal IP beam size

\section{OCTUPOLE CORRECTION.}

Once the $y^{\prime 2} \delta^{2}$ term has been cancelled, the minimum $\sigma^{*}$, is achieved with a $\beta^{*}{ }_{y} \sim 2 \mathrm{~mm}\left(\theta^{*}{ }_{y}-180 \mu \mathrm{r}\right)$, with a reduction in beam size of approximately 1.8 . The remaining aberrations are the octupole terms arising from the interaction of the interleaved sextupole pairs, and to some extent the long sextupoles themselves. Table 2 summarizes the remaining important aherrations for the new linear optics. These terms can be corrected by the addition of octupoles. The chromatic sextupole term $\left(x^{\prime} y^{\prime 2} \delta\right)$ can be removed by placing a -I pair of octupoles in the $\mathrm{CCS}$. This pair, when powered anti-symmetrically generate the required $x^{\prime} y^{\prime 2} \delta$ term. together with $x^{\prime 3} \delta$ and $x^{\prime} \delta^{3}$ terms which affect the horizontal IP beam size. When powered symmetrically, the pair generates pure geometric octupole ternis $\left(x^{4}\right.$, $\left.x^{\prime 2} y^{\prime 2}, y^{4}\right)$, together with the high order chromaticity terms $x^{\prime 2} \delta^{2}$ and $y^{\prime 2} \delta^{2}$ (the very terms that the final telescope modifi- 


\begin{tabular}{cccc}
\hline Monomial & $\begin{array}{c}\text { TRANSPORT } \\
\text { notation }\end{array}$ & $\begin{array}{c}\text { Coefficient } \\
\text { (meters) }\end{array}$ & $\begin{array}{c}\text { \% of total } \\
\sigma^{2} y\end{array}$ \\
\hline$x^{\prime} y^{\prime 2} \delta$ & $\mathrm{U}_{1446^{\prime}} / \mathrm{U}_{3246}$ & -858.4 & 50 \\
linear & - & - & 21 \\
$x^{\prime 2} y^{\prime 2}$ & $\mathrm{U}_{1244} / \mathrm{U}_{3224}$ & -3104.8 & 20 \\
$y^{4}$ & $\mathrm{U}_{3446}$ & 55.5 & 9 \\
\hline
\end{tabular}

Table 2. Most significant aberrations to $\sigma^{*}$ in order of contribution for new final telescope optics $\left(\theta^{*}=245 \mu \mathrm{r}\right)$.

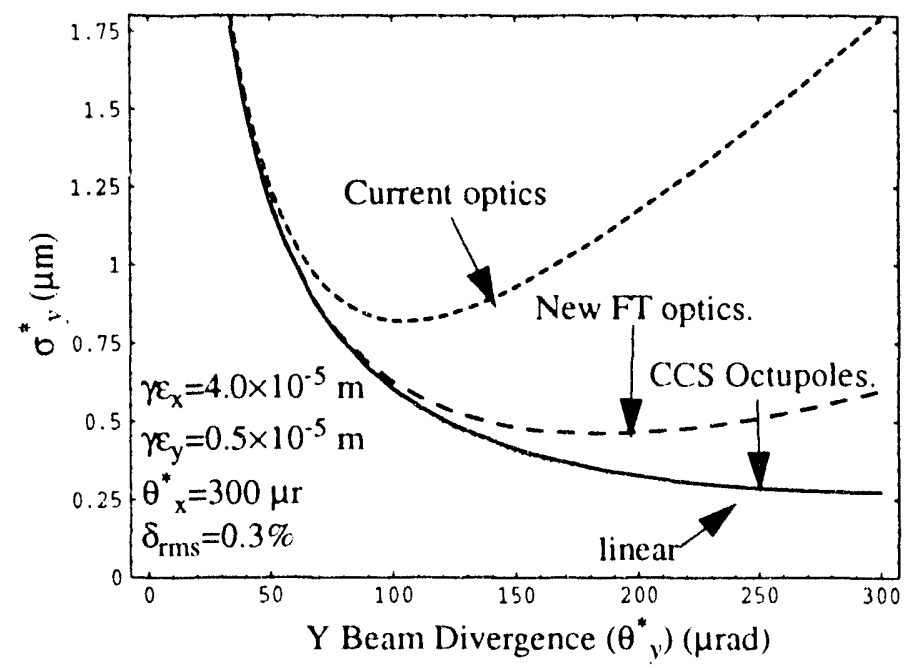

Figure 2. Vertical IP heam size $\left(\sigma^{*}\right)$ as a function of vertical IP beam divergence $\left(\theta^{*}\right.$ ) for the current optics, final telescope modifications and CCS octupole compensation.

cations are designed to cancel). One possible solution is to place an anti-symmetric -I pair in the CCS to cancel the dominant $x^{\prime} y^{\prime 2} \delta$ term, and use three independent octupoles in the final telescope to exactly cancel the three pure geometric terms. The location of the -I CCS octupole pair is governed by (a) the maximum realizable octupole strength and (b) the tolerance on the $x^{3} \delta$ term. The five octupole solution effectively reduces the vertical spot size to the linear limit with a divergence of $245 \mu \mathrm{r}$. A cheaper two ociupole solution, which still uses a -I CCS pair, has been adopted with only a small loss in luminosity. The two octupoles are now run unequally, so as to reduce the RMS of the contributions of the various aberrations listed above. The solution was arrived at by first adjusting the octupole strengths anti-symmetrically to zero the $x^{\prime} y^{\prime 2} \delta$ term, and then symmetrically to minimize the pure geometric terms (the high order chromaticity terms generated being negligibly small). The solution obtained resulted in the downstream octupole having an integrated strength of $\sim 900 \mathrm{~m}^{-3}$, while the upstream octupole runs at approximately half that value. achieving a vertical beam size of $340 \mathrm{~nm}$. Figure 2 shows $\sigma^{*}$, as a function divergence for the proposed improvements.

\section{NEW TUNING STRATEGY.}

The current SLC final focus design utilizes two magnets in the upper telescope (UT), together with the final triplet and a small tuning quadrupole to adjust the $\beta^{*}$. To maintain a static optics in the CCS and final telescopes (with the exception of small adjustments for fine tuning), it is proposed to move all the beam matching to the UT. To facilitate this, a new wire scanner capable of resolving a $1.5 \mu \mathrm{m}$ vertical beam size will be installed at the IP image point at the entrance of the CCS. In addition, a new quadrupole will be installed in the UT 10 allow for a global tuning algorithm[6]. A new skew-quadrupole will also be installed to control the $\langle x y\rangle$ correlation in the heam. It is also proposed to include several new wire scanners throughout the UT and $\eta$ suppression section to allow fast measurement of the incoming beam phase space[7].

\section{PROJECTED LUMINOSITY GAIN.}

Table 3 gives the expected IP vertical beam size, luminosity (in units of $\mathrm{Z}$ particle production per hour) and beam-beam enhancement for the current final focus and the two proposed improvements. The enhancement factor $\left(H_{D}\right)$ is calculated from scaling laws[8] and is strongly dependent on bunch length and bunch current. For all three scenarios, the horizontal beam size remains roughly constant at about $2 \mu \mathrm{m}$.

Table 3: Expected vertical heam size and luminosity for the current final focus and the two proposed improvements. Other heam paramcters factored into the calculations are: $\varepsilon_{\lambda}=6(x) \mu m \mu r$, $\varepsilon_{y}=6() \mu \mathrm{m} \mu \mathrm{r}, \theta_{x}^{*}=3(x) \mu \mathrm{r}, \sigma_{z}^{*}=0.8 \mathrm{~mm}, \delta_{m . s}=0.3 \%$, $N_{b}=3.5 \times 10^{70} \mathrm{pph}$.

\begin{tabular}{cccccc}
\hline & $\begin{array}{c}\theta^{*}{ }_{y} \\
(\mu \mathrm{r})\end{array}$ & $\begin{array}{c}\sigma^{*} \mathrm{y} \\
(\mu \mathrm{m})\end{array}$ & $\begin{array}{c}L \\
(\mathrm{Z} / \mathrm{hr})\end{array}$ & $\mathrm{H}_{\mathrm{i})}$ & $L^{*}=\mathrm{H}_{1} L$ \\
\hline Current FF & $1(14$ & 0.839 & 66 & 1.16 & 76 \\
Fr Upgrade & 190 & 0.462 & 122 & 1.41 & 171 \\
FT Upgradet & 245 & 0.340 & 170 & 1.44 & 2.45 \\
CCS Oclupoles & & & & & \\
\hline
\end{tabular}

\section{REFERENCES}

[1] K. Brown, "A Conceptual Design of Final Focus Systems for Linear Colliders", SLAC-PUB-4159, (1987).

[2] J. Murray, K. Brown, and T. Fieguth, "The Completed Design of the SLC Final Focus System", SLAC-PUB4219 (1987).

[3] N. Walker, J. Irwin, and M. Woodley, "Analysis of Higher Order Optical Aberrations in the SLC Final Focus using Lie Algebra Techniques.", these proceedings (1993).

[4] C. Adolphsen et al, "Flat Beams in the SLC", these proceedings (1993).

[5] K. Brown, "A First- and Second-Order Matrix Theory for the Design of Beam Transport Systems and Charged Particle Spectrometers", SLAC-75 or Advances in Particle Physics 1, 71 (1967).

[6] N. Walker, J. Irwin, and M. Woodley, "Global Tuning Knobs for the SLC Final Focus", these proceedings (1993).

[7] P. Emma, private communication.

[8] P. Chen, and K. Yokoya, Phys. Rev. D38, 987 (1988). 

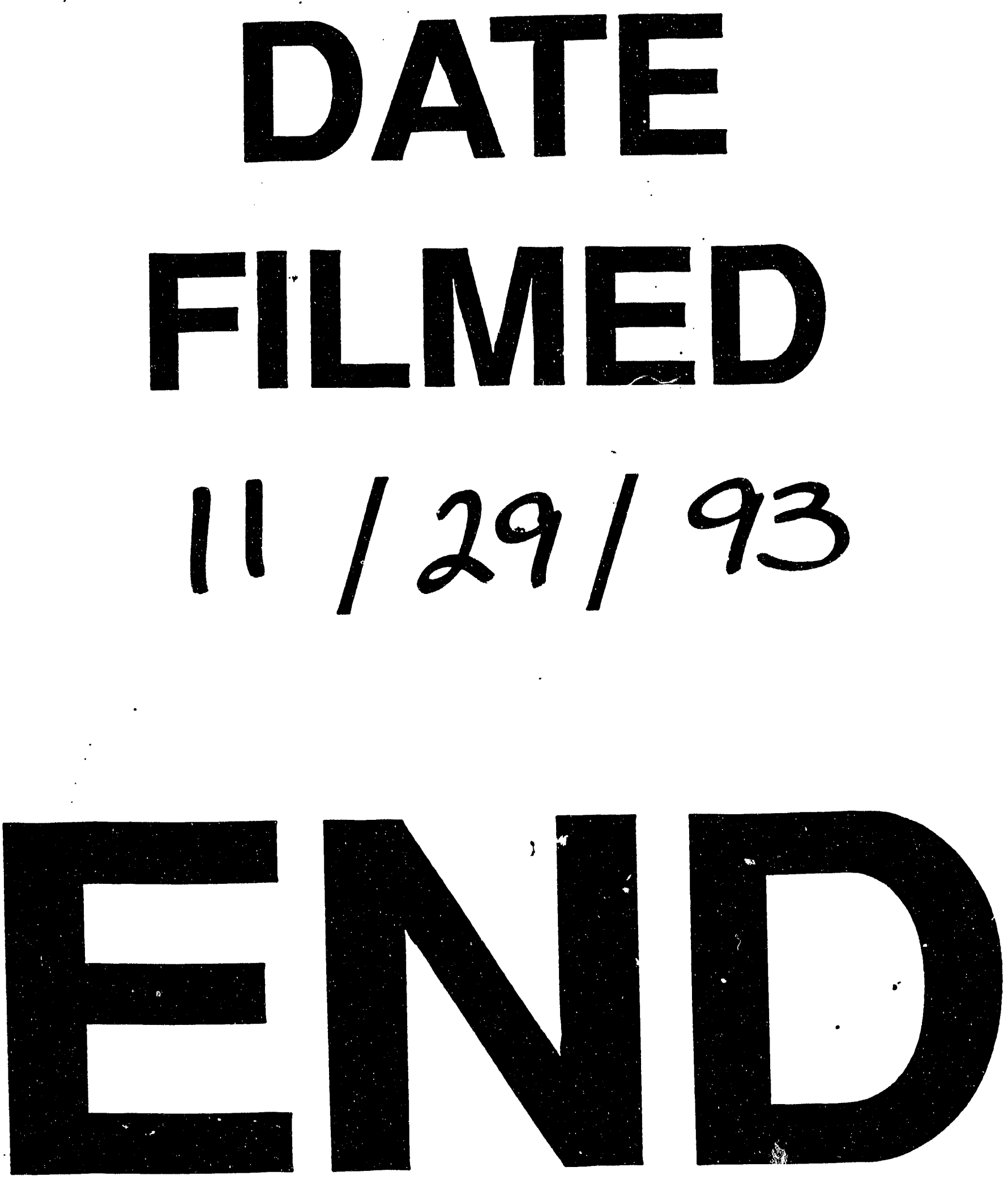
